'It looks good on paper': Transitions of care between midwives and child and family health nurses in New South Wales

Caroline SE Homer ${ }^{\mathrm{a}}$

Professor of Midwifery

Kathryn Henry ${ }^{\mathrm{a}}$

Research Midwife

Virginia Schmied ${ }^{\mathrm{b}}$

Associate Professor (Midwifery/Child and Family Health)

Lynn Kemp ${ }^{\mathrm{c}}$

Senior Research Fellow

Nicky Leap ${ }^{\mathrm{a}, \mathrm{d}}$

Professor of Midwifery Practice Development and Research

Carolyn Briggs ${ }^{\mathrm{a}}$

Senior Lecturer

\title{
Affiliations
}

${ }^{a}$ Centre for Midwifery, Child and Family Health, Faculty of Nursing, Midwifery and Health, University of Technology Sydney

${ }^{\mathrm{b}}$ College of Health Sciences, University of Western Sydney

${ }^{\mathrm{c} C e n t r e ~ f o r ~ H e a l t h ~ E q u i t y ~ T r a i n i n g ~ R e s e a r c h ~ a n d ~ E v a l u a t i o n, ~ U n i v e r s i t y ~ o f ~ N S W ~}$

${ }^{\mathrm{d}}$ South Eastern Sydney Illawarra Area Health Service 


\section{'It looks good on paper': Transitions of care between midwives and child and family health nurses in New South Wales}

\section{ABSTRACT \\ Background}

The way in which women and their babies transition from maternity services to the care of child and family health nurses differs across Australia. The aim of the study was to understand the transition of care from one service to another and how to promote collaboration in the first few weeks after the birth.

\section{Method}

A descriptive study was undertaken. All midwifery, child and family health and Families NSW managers in NSW were invited to participate by completing a questionnaire.

\section{Results}

There was a wide range of transition of care models. These varied by setting, geography, context and history. Three main models emerged from the analysis. These were a:

1. Structured, non-verbal communication system that relied on paper-based or computerised systems. This included either centralised referral or centre-based referral processes

2. Liaison person model which was similar to purposeful contact, but with everything vested in one clinician who is responsible for the coordination and organisation.

3. Purposeful contact model which was mostly for identified at-risk women and included continuity of care with formal networks and face to face contact.

\section{Discussion}

There were a range of different models of transition of care identified in NSW depending on local context, expertise, interests and policies. Some are very structured and others have developed and evolved over time. Many models seem to be dependant on the goodwill and enthusiasm of individual clinicians. 


\section{Conclusion}

A more coordinated and systematised approach needs to be developed. Collaboration and communication between midwives and child and family health nurses is essential if the needs of families are to be addressed during this transition period.

Keywords: midwives, maternity services, community child health, child and family health nurses, health visitors, maternal and child health

Word Count: 4700 


\section{'IT LOOKS GOOD ON PAPER': TRANSITIONS OF CARE BETWEEN MIDWIVES AND CHILD AND FAMILY HEALTH NURSES IN NSW}

\section{INTRODUCTION}

Currently, the way in which women and their babies move through the health system from maternity to child and family health nursing ${ }^{1}$ services differs widely around Australia. Little is understood about the capacity to transfer care effectively from one service provider to another or how to promote collaboration within the current systems in the first few weeks after birth. There is also little knowledge of how best to use services and integrate these to meet the needs of families during this transition period. This study aimed to improve understanding of the most effective strategies for transition of care for childbearing women from midwifery to child and family health nursing services.

The pregnancy and early childhood period provides a 'window of opportunity' in which to assist families to access services and support. Australia has a well-established system of universal health services directed at meeting the needs of pregnant women, infants, young children and families and is provided at multiple contact points. Almost all women will receive the care of a midwife at some time during pregnancy, labour and birth and/or the postnatal period. Australia also has a system of free, universal services for children from birth to the age of five years, provided by community-based child and family health nurses.

Transition of care from one service to the other generally occurs in the first two weeks after birth but can be up to eight weeks later. This is an ideal time to ensure that women are linked into services and support agencies during the early childhood period to assist their transition to parenting. This is also the time that fragmentation of services can potentially have a deleterious effect [1]. Consequently, Australia's National Agenda for Early Childhood and numerous state-based policies [2,3] are premised

\footnotetext{
${ }^{1}$ Child and family health nurse may also be called a maternal and child health nurse, child and youth health nurse or child health nurse in different states/territories.
} 
on a model of coordination and integration of care for families. The role of both midwives and child and family health nurses within such initiatives has not been explored fully. The potential of midwifery, as well as child and family health nursing, as a public health strategy is often not understood in Australia as clearly as it is in other countries [4,6].

There is very little written about the relationship and integration of child and family health nursing and midwifery services. The Victorian Department of Human Services has published a specific document Continuity of Care [7] which outlines the process of the way in which women should transitioned from maternity services to maternal and child health services and a standardised approach to the transfer of information between these services across a variety of settings. Integration literature suggests that improved coordination of care makes a difference in terms of effective support for new mothers and families, particularly those with additional needs for psychosocial support [8]. Currently in Australia, this level of coordination is not the case [1]. The impact of the fragmentation of the health system on health service delivery and health outcomes has been well documented [9] and numerous attempts are being made at a service level to address these concerns. The focus of activities in recent times has been on the vertical integration of primary and community health services with specialist shared care programs, discharge planning and post-acute care [10]. However, much less attention has been paid to horizontal, overlapping integration across care providers to develop a more coherent service delivery, particularly in the areas of preventive care, early detection and intervention [11] which is likely to be beneficial. In developed countries, maternity care provision is also moving towards a primary care model with an emphasis on community-based services, partnerships and collaboration [12]. Little is understood about the impact of fragmentation on the capacity to transfer care effectively from one service provider to another in the perinatal period.

Collaboration between organisations which provide services to women, children and families is paramount to an effective and smooth delivery of care and satisfaction of caregivers and families. Collaboration across traditional service boundaries and integration of service delivery potentially results in, enhanced access to services; improved health outcomes; a wider choice for consumers; and, 
a reduction in the use of inappropriate or unnecessary services $[13,16]$. This does not always occur and there are complex professional and organisational boundaries that create barriers to relationships and effective working practices $[17,18]$. One example is when midwives and child and family health nurses (CFHN) are often organised under separate management structures which does not promote effective communication and collaboration. .

Anecdotally, professional tensions have been seen to occur between midwives and child and family health nurses. These may inhibit collaborative approaches and lead to ineffective transition of care for families. This can often simply be a lack of appropriate communication over concerns about particular families (for example, communication that is limited to a brief fax) which may further the separation between the two groups. It could also be that, problems with communication arise because midwives and CFHNs rarely meet. Most work in different locations, do not regularly attend in-service education initiatives of common interest together (despite their roles overlapping in the early postnatal field) and rarely have face-to-face meetings. There are also possible conflicts over management strategies (for example, around breastfeeding) initiated by midwives that may differ from those used by the CFHNs. Furthermore, the timeframe of care overlaps and this can add to the tensions of 'who is caring for the family'. As identified by the World Health Organization, the midwifery role extends to the end of the postnatal period, six weeks following birth and includes home visiting in the community [19]. During this window of time there is the potential for effective dovetailing of services with CFHNs who are also involved in home visiting and whose role in supporting families can extend for many years.

Despite initiatives in many Australian states and territories, which aim to develop a coordinated network of services for families and a system of providing appropriate support, anecdotal experience and recent government evaluations [20] suggest that systems vary across New South Wales (NSW). In NSW, there are diverse ways in which midwives transfer care to CFHNs. Concerns have been expressed to the authors that the diversity could be problematic as it means there is a lack of consistency. 
The aim of this study, therefore, was to examine the characteristics and nature of effective transitions of care in NSW between midwives and CFHNs. The specific objectives were:

- Describe the current approaches to transitions of care from midwives to CFHNs; and

- Understand the barriers and facilitators to effective transition of care.

The study was approved by the University’s Human Research Ethics Committee prior to commencement.

\section{METHODS}

A descriptive study was undertaken using a questionnaire for managers and clinical leaders from each of the Area Health Services (AHS) in NSW. There are currently nine AHSs in NSW with five to ten ‘clusters’ or smaller areas within each. Most of the clusters have a manager or clinical leader responsible for midwifery and/or child and family health services. There are also Families First (now called Families NSW) Coordinators, who are based in each AHS. These coordinators are responsible for implementation of the NSW strategies, which include universal home visiting, usually by CFHNs.

Initially, a questionnaire that had previously been used in integration studies with general practitioners (GPs) was adapted [21] for this study. This questionnaire used a quantitative approach and required respondents to identify their type of service from a structured list. A focus group with midwives was undertaken using the adapted questionnaire to determine its acceptability and utility in the context of midwifery and child and family health services. The adapted questionnaire was found to be inadequate and inappropriate for achieving the aims of the study as it could not account for the wide diversity of the models of care. The recommendation from the focus group was that a more open-ended or qualitative approach be taken.

Subsequently, a series of open-ended questions were developed for the questionnaire. These were pilot tested with two focus groups - one with members from the NSW Midwives Association (NSWMA) 
and one with members from the Child and Family Health Nurses Association NSW (CAFHNA). This revised approach, which collected qualitative rather than quantitative data, was seen to be more acceptable and appropriate than the initial approach.

The questionnaire included two demographic questions (identification of AHS and respondent's role) and then a series of open-ended questions that asked about the:

- Pathways of transition from midwifery to child and family health services;

- Factors that facilitate effective transitions of care between midwives and child and family health nurses;

- Potential or real barriers to effective transitions of care;

- Characteristics of an ideal pathway for the most effective transition of women and their babies from midwifery to child and family health services.

Respondents were also invited to send a diagram mapping how services intersect and showing the pathway taken by new mothers.

In order to identify the sample, we attended the routine state-wide meetings of the managers of Child and Family Health Services; the Clinical Nurse Consultants for child and family health; and the managers of Families NSW. We contacted the NSW Midwifery Consultants Group to identify the midwifery leaders and used the NSW Pregnancy and Newborn Services Network email list to access all maternity managers. These various sources provided a distribution list of 81 managers and consultants.

An email inviting participation was sent to the identified managers and clinical leaders $(n=81)$. This informed potential participants of the purpose of the study and invited those who wished to participate to respond to the attached questionnaire by email, fax, or postage paid envelope. The response to the email invitation was rapid, with the majority of questionnaires returned within approximately four weeks. Email contact was chosen to reduce the time lag between traditional postal options, it is low 
cost; promotes data collection efficiency; and has potentially higher response rates through rapid communication with participants.

Data were transcribed into an Excel spreadsheet and analysed using content analysis [22]. Key themes were identified by all members of the five research team working in a group. This group process assisted trustworthiness of the analysis and ensured that all results were thoroughly discussed with the team.

\section{RESULTS}

There were 67 responses to the questionnaire (response rate 83\%). The respondents were from each of the nine AHS and included a similar proportion of midwives and CFHNs (Tables 1 and 2).

\section{Models of transition of care}

There was a wide range of transition of care models identified from the 67 respondents. These varied by setting, geography, context and history and whether women and families were identified as 'low risk' or 'medium to high risk'. The concept of 'risk' seemed to be a broad one with considerable emphasis on social and emotional risk and other markers of vulnerability (eg. adolescence). One third of respondents $(\mathrm{n}=22)$ also provided a diagram which graphically outlined the pathways that women undertook. Some of these diagrams were very detailed and showed evidence of considerable planning and consultation, while others were simple. The diagrams were evidence of how local settings had operationalised the process.

Within each AHS, numerous models co-existed, often due to historical reasons (for example, there used to be a different AHS before restructuring in 2005/6) or the identified needs of the local community (for example, those of Indigenous communities). Three main models emerged, although 
these were not mutually exclusive and some respondents identified more than one approach. The main models were:

1. Structured non-verbal communication $(n=45)$

a. centralised referral process $(n=8)$

b. centre-based referral process $(n=37)$

2. Liaison person $(\mathrm{n}=17)$

3. Purposeful contact $(\mathrm{n}=26)$

Each of the main models will be described with illustrative quotes or examples taken verbatim from the responses. In some instances, additional text has been added in square brackets to aid readability. Names and AHSs have been deleted for confidentiality.

\section{Structured non-verbal communication}

Structured non-verbal communication was the most common approach to transition of care. In total, 45 respondents identified this approach (eight described a centralised intake process and 37 identified the centre-based referral process).

The centralised intake model was reported from three AHSs, (although not all facilities within the these AHSs were using this approach). Respondents described the approach as:

The mother is informed of her local child and family health centre by the maternity staff on discharge (most of the time). She is then advised to call the child and family health centre to make an appointment for her first visit. The obstetric summary is faxed to the child and family health centre via the [central place] (Manager of CFHN).

All obstetric discharge summaries are sent to a central registration officer who forwards them on to the relevant child and family health centre (Clinical leader - CFHN).

In other cases, the discharge summary was electronically transferred to the centralised intake place for allocation to the relevant child and family health centre. Sometimes the centralised intake approach was combined with a liaison role. For example: 
In our sector the [computer generated] discharge summary is forwarded by either the Community Liaison Midwife or by the discharge planner of the hospital to our Centralised Intake for all women delivering in local hospitals (Clinical leader - CFHN).

The centre-based referral process was more widespread, being reported from eight of the nine AHSs. Women are provided with information to enable them to make their own follow-up arrangements with the CFHN. Discharge summaries are faxed or mailed to each woman's local child and family health centre (nearest to her home). Typical examples include:

The majority are via [the discharge summary being sent] to CFHN in area of residence (Clinical leader - midwife).

Following the birth of baby, the discharge summary is sent to the child and family services (on daily basis) - phone contact [is] made with women by the CFHN and [a] home visit or appointment arranged (Manager - midwifery).

\section{Liaison person}

The liaison or discharge planner model places the responsibility for identification and communication on one person. The model is predicated on having a person (midwife or CFHN) who undertakes to see or review the records of each postnatal woman while in hospital or still receiving midwifery care at home and determine the ongoing follow-up and referral. Overall, 17 respondents identified this approach.

An example of this model was:

A summary of the maternal discharge form and neonatal discharge form is given to the early childhood liaison officer. She also does rounds of the postnatal ward and collects forms and sees all the women on the ward. She explains the early childhood services and where the woman's nearest centre is. She then puts a sticker (included) on the blue book with the name and address of the centre (Clinical leader - midwife). 
These liaison roles generally have limited hours attached for this process so alternative approaches also exist:

New mothers in the hospital are given the information regarding where the nearest early childhood health service ${ }^{2}$ is to where she lives via the community liaison nurse. [The] position works Monday to Friday. If mothers have not been seen by the community liaison nurse then the midwives give the information of the nearest early childhood health centre and how to go about making contact (Manager - CFHN).

\section{Purposeful contact}

Purposeful contact was a model used mostly for identified at-risk women and included continuity of care with formal networks and face to face contact. Overall, 26 respondents identified this approach and it was often used in tandem with the structured non-verbal communication model and the liaison model. The strategies used included, the establishment of formal networks with regular meetings; verbal referral and handover; joint case management and, feedback.

A number of examples were provided by respondents:

High risk women - complex cases are identified and flagged at [the] booking visit and a multidisciplinary meeting plans for women and discharge planner links women to [the] appropriate service (Clinical leader - midwife).

Women or babies who are identified as being at risk either antenatally/ postnatally are flagged by the staff and at a postnatal multi-disciplinary meeting held weekly, and are then refereed to the Parenting Support Service (Clinical leader - midwife).

[there is] specific referral from [the] Maternity Unit to Child and Family Health Nursing if the family has vulnerabilities or risk factors (Manager - CFHN).

\footnotetext{
${ }^{2}$ Early Childhood Health Centre is the older term for the Child and Family Health Centre and is still used by many of the respondents in the study
} 
Some respondents stated that this approach was seen as beneficial but was not always carried through. For example:

The midwives in the Maternity Unit may also ring the CFHNs and make a referral over the phone. Unfortunately this does not happen as much as it should (Clinical leader - CFHN).

These models worked well when the providers had clearly defined roles (for example, who was going to provide care and which aspects of care would be provided) and there was multidisciplinary leadership. There were also examples of the model being person-dependant. For example, a perinatal mental health worker may facilitate the multidisciplinary meetings but if that person left the role, meetings failed to happen.

\section{Other models}

Two less common models were identified. These were an unstructured model; and, shared visits (midwife and CFHN) in the postnatal period.

The unstructured, and perhaps more flexible model, is generally when services (midwifery and CFHN) are co-located and informal communication is easier to facilitate. Only two respondents clearly identified such a model, both based in rural settings. In one case, the role of community midwife and child and family health nurse was held by the same person and in the other, collaborative working occurred in a small rural hospital:

We are a small rural hospital, with a small number of midwives and one full time CFHN and one part time [CFHN] - because it is a small community there is good communication between the two services (Clinical leader - midwife).

A shared visit in the postnatal period by the midwife and CFHN was highlighted by three respondents as a model of care that was appropriate and effective for women who have a high level of social and emotional need or vulnerability. This sharing of visits was often unstructured and dependant on the context and the needs of specific women. Six respondents stated that antenatal referrals were made to 
child and family health nurses and women were provided with information about child and family health nursing services in the antenatal period. Only one respondent reported that CFHNs were involved in visits during the pregnancy.

For the most part, the models of transition were inconsistent across area health services. Specific models were not mandated by the area health services, except in one area that had a centralised referral process. Many of the models were adhoc and had developed according to local need and context rather than a systematic approach.

\section{Facilitators and barriers}

There were 91 comments about the facilitators of effective transition of care and 88 comments about the barriers. The most highly cited facilitators were, effective communication which included regular meetings and shared education ( $\mathrm{n}=25$ ); having mechanisms that built relationships between midwives and child and family health nurses, including the CFHN visiting the maternity unit regularly, encouraging verbal handover, using similar assessment tools and being co-located $(\mathrm{n}=28)$; having a central intake point or a designated person, especially for women from vulnerable groups $(n=13)$ and, having completed up to date discharge summaries including the correct address and telephone number for the woman $(\mathrm{n}=10)$.

The following quote illustrates the need for relationships and communication to facilitate transition: A major contribution to a smooth transition of care for parents from the midwives to the CFHNs is a good working relationship between both services. This is facilitated by trust and regular meetings (second monthly) and any difficulties in communication can be addressed face to face at these meetings ... team building is absolutely essential in large organisations. Some of these meetings need to allow time for networking, socialisation and 'cuppa tea' to release these frustrations (Clinical leader - CFHN). 
There were 88 comments about the barriers to effective transition of care. The most common responses were: lack of staffing, including the casualisation of the workforce and the removal of some community midwifery and nursing positions in some AHSs $(n=22)$; the delay in CFHNs receiving the discharge information from the maternity unit ( $\mathrm{n}=17)$; a lack of understanding of, and respect for, one another's roles and areas of expertise $(n=10)$; women's lack of knowledge about the role of the CFHN $(n=8)$; and a lack of information technology infrastructure, especially for CFHNs ( $n=7)$.

Professional boundaries and poor communication were highlighted as significant issues as typified in these quotes:

[There is] a misunderstanding between midwives and CFHN of each other's expertise / work practices and appropriate transition timeframes (Families NSW coordinator). [There is a] lack of understanding [from midwives as to] what happens to a client when they are discharged and the transition into parenting and the pressures on parents that result" (Clinical leader - CFHN).

[There is a] lack of understanding of the role of CFHN. We have been up there on numerous occasions to give in-services and few midwives turn up (Clinical leader - CFHN). The holding back of information that is either relevant for the client's care, such as, mental or drug health issues, or information regarding safety, such as domestic violence, is not always passed on to the Child and Family Health Nurses (Manager - CFHN).

[there is] poor communication between the maternity unit and child and family health services. We often find that mothers turn up to our service for a first visit without any initial contact with us and with multiple risk factors ... the maternity unit either does not communicate with our staff or the staff within the maternity unit do not communicate with each other (Manager - CFHN).

Most of the concerns were about a lack of understanding of one another's roles, for example: [There is] ignorance of how the two systems work. The two areas of Maternity and CFHN have been traditionally seen as two separate entities (Manager - midwifery). 
There was also a lack of understanding about how midwifery and CFHN services could co-exist in the early postnatal period, typified by:

Where good midwifery services are available postnatally, this can actually reduce women's access to CFHN. There can be disputes over who the client belongs to and confusion for the women about which nurse has contacted them for an appointment. Women are promised an offer of a home visit by a CFHN within two weeks, however if a midwife is still attending the home this can be unnecessary, confusing and even distressing for the women (FF Coordinator).

As highlighted earlier, the transfer of information was both a facilitator and a barrier to effective transition. The specific mechanisms in which to transfer information depended on the type of model and the systems (including access to computers) available in the sites. Some settings transferred information through fax (eg. centre based referral model) or electronically (centralised intake model). Some models transferred information antenatally especially for women considered to be at risk, whereas many others transferred information only after the birth of the baby. The type of information also varied although for most women it was limited to birth data and any identified problems.

\section{DISCUSSION}

This study identified a diversity of models and pathways of transition from midwifery to child and family health services. While three main models have been identified, numerous adaptations and combinations were in place across the state both for women with and without identified risk factors. At times these seemed adhoc and lacked consistency. The majority of transition models are based on a non-verbal approach with often little contact between the services and the individuals. This limited contact limits the understanding about the roles and the contribution that each can make. The results highlight the need for better communication and relationship-building between the two groups. 
When women are identified as having high needs, a purposeful model is often used. This is predicated on women actually being identified as having a high level of need (usually this is social and emotional rather than physical) and having services that can address the needs. In NSW, this has largely been influenced by the Integrated Perinatal and Infant Care Initiative [23] which aimed to encourage integration and collaboration across health and related services.

The liaison model was identified by around one fifth of the respondents. This is a useful model, however it has limitations. Having such a model is predicated on having adequate resources to support the role of a person responsible for liaison. Most of the examples given reported that this person was available only during office hours or on certain days of the week. Some of the positions were not replaced when the incumbent was on leave. In this model, all the information and knowledge about the services available for women in the community are vested in one person and this means that others (particularly hospital-based midwives) have limited knowledge of the resources and services available. The role also contributes to, fragmented care; women have yet another professional to 'tell their story to' and this can mean that information is omitted or misunderstood. Nonetheless, this role can be useful as a point of central coordination, particularly for women from vulnerable or marginalised groups.

There seems to be a lack of formal mechanisms that promote collaboration and communication between midwives and child and family health nurses. The management structure and accountability and reporting lines are often totally separate with limited formal data linkage processes, this exacerbates the fragmentation of services. The lack of understanding from both professional groups about their defined sphere and scope of practice and how these might dovetail contributes to a lack of collaboration. Disagreements over professional boundaries and who should be providing home visits have meant transitions of care have not always been smooth.

Importantly, new policy directions [24,25] and evidence from studies of child and family health nursing practice [26], indicate that contact with a family during the pregnancy can facilitate 
engagement with services following birth [27]. Recent policy approaches suggest that CFHNs should become involved in the care of, and support for, families before birth, particularly disadvantaged families although this has not always been easy to implement in practice [28]. It is suggested in at least one of the recent policies [24] that the final midwifery visit to a family should be jointly with the CFHN. A joint visit can facilitate trust between services and assist the transition for families.

The principle of a primary health care approach [29] is essential, both for midwifery and child and family health services. Child and family health nurses are more familiar with this philosophical approach as they have always been based in the community and have been informed by principles of primary health care since the 1980s [28]. The NSW competency standards for CFHNs clearly identify primary health care as a key principle of practice [30]. Midwives are only starting to understand that this is a fundamental part of their role, even if they have only ever been based in hospitals [31]. In recognition of this, the National Competency Standards for the Midwife have, 'Midwifery as primary health care' as one of the four domains [32]. The standards explicitly state that midwifery has a "primary health care framework and is committed to seeing midwifery as a public health strategy that encompasses a broad social context” [32, p.2].

Continuity of care was an important facilitator and a principle of effective transition. The definitions of continuity are broad and often confusing. One way to consider continuity is to define it as a hierarchical concept, ranging from the basic availability of information about the woman's past history to a complex interpersonal relationship between provider and woman, characterised by trust and a sense of responsibility. This concept is outlined in Figure 1, based on the work of Saultz and colleagues [33,35]. Understanding continuity as a hierarchical concept may be a useful approach when considering effective transition from midwifery to child and family health services.

The study is limited by a number of factors. The sample may not be representative of all midwifery and child and family health services in NSW. Attempts were made to ensure a wide range of managers, clinical leaders and coordinators were invited to participate. The response rate of $83 \%$ is 
encouraging, as is the response from each AHS in the state. The complexity of the models of transition across the state meant that it was impossible to develop a quantitative questionnaire that would have enabled responders to explain their systems fully within a comprehensive framework, allowing quantitative comparisons. The qualitative nature of the data allows only for content analysis. The study was also unable to determine exactly what 'best practice' should be and how the transition should occur. Services should be developed in line with policy direction and general principles that place women, children and families at the centre of care. This paper has highlighted general principles that should be considered in the development of best practice models but the actual service will vary according to local need and context.

The NSW public health system has been going through a period of restructure in the past two years. This means that many services and processes are changing as a result of AHS amalgamation. The time delay from the completion of the questionnaires (late 2006) and the publication of the results may mean that aspects of the models have changed in that time. The study is also limited in that it only canvassed the views of health professionals. It is important in future research in this area to study the experiences of women and their families in the transition of care process. Finally, these findings are based on the experiences of health professionals within the public health sector in one Australian state but are likely to have resonance in other states and territories and in similar countries.

\section{CONCLUSION}

The study has highlighted the diversity of models of transition of care from midwifery to child and family health services in NSW. Many of the models of transitions of care were 'bandaid' options that grew over time, were not coordinated, and were dependant on the goodwill and enthusiasm of individuals.

A more coordinated and systematic approach needs to be developed. Collaboration, communication and mutual respect between midwives and CFHNs is essential, especially in light of the developing roles of the two disciplines. Flexibility and local models for local contexts should continue to be 
important principles, however, a more coordinated or integrated approach is needed if the needs of new mothers are to be addressed. Future research also needs to consider the views and experiences of women and their families in negotiating transition of care as well as other issues such as the timing of information transfer, the quality of information; the way the profession can better collaborate; the importance of continuity of care for women with complex problems; and the effectiveness of child and family health nurses engaging with pregnant women. 


\section{REFERENCES}

1. Brotherhood of St Laurence. Breaking Cycles, Building Futures. Promoting inclusion of vulnerable families in antenatal and universal early childhood services. 2005 [cited 2004 10th July]; Available from: http://www.beststart.vic.gov.au/docs/ecs_breaking_cyclesbest_start.pdf 2. Queensland Health. Midwifery models of care: Implementation guide. Brisbane: Queensland Health; 2008.

3. WA Health Department. Improving Maternity Choices: Working Together Across WA. Perth: Health Policy and Clinical Reform, WA Department of Health; 2007.

4. Kaufman K. Commentary: Have we yet learned about the effects of continuity of midwifery care? Birth 2000; 27(3):174-6.

5. Kaufman KJ. Effective control or effective care? Birth 1993; 20(3):156-8.

6. Kaufmann T. Policy. Life after birth: reprioritising postnatal care. RCM Midwives Journal 2000; 3(11):338-9.

7. Victorian Department of Health. A communication protocol for Victorian public maternity services and the Maternal Child Health Service. Melbourne: Victorian Government; 2004.

8. Rodríguez C, des Rivières-Pigeon C. A literature review on integrated perinatal care. International Journal of Integrated Care 2007; 7(e28):1-15.

9. Fry D, Furler H. General practice, primary health care and population health interface. General Practice in Australia. Department of Health and Aged Care, Commonwealth of Australia, Canberra; 2001.

10. Harris M, Powell Davies G. Integration between GPs, hospitals and community health services. General Practice in Australia. Commonwealth of Australia, Canberra: Department of Health and Aged Care; 2000.

11. Briggs CJ, Capdegelle P, Garner P. Cochrane Database of Systematic Reviews. 2005;1:1.

12. NSW Health. A Framework for Managing the Quality of Health Services in NSW. State Health Publication No: (HPA) 990024. Sydney: NSW Health Department; 2000. 
13. Katz I, Hetherington R. Co-operating and communicating: A European perspective on integrating services for children. Child Abuse Review 2006; 15(6):429-39.

14. Huxham C, Vangen S. Doing things collaboratively: Realizing the advantage or succumbing to inertia. Organizational Dynamics 2004; 33(2):190-201.

15. Leutz WN. Five Laws for Integrating Medical and Social Services: Lessons from the United States and the United Kingdom. Milbank Quarterly 1999; 77(1):77-110. 1999.

16. Frost N, Robinson M. Joining up children's services: safeguarding children in multidesciplinary team. Child Abuse Review 2007; 16(3):184-99.

17. Ellefsen B. The experience of collaboration: a comparison of health visiting in Scotland and Norway. International Nursing Review 2002; 49(3):144-53.

18. Hall P. Interprofessional teamwork: Professional cultures as barriers. Journal of Interprofessional Care. 2005; 19(Supplement 1):S188-S96.

19. WHO Technical Working Group. Postpartum care of the mother and newborn: A practical guide. Birth 1999;26(4):255-8.

20. Valentine K, Fisher K, Thomson C. Making integration happen: the Families First policy experience. Child Abuse Review 2006;15:414-28.

21. Reynolds F, Oldroyd J, Harris M, Powell Davies G. More bridges: the continuing impact of General Practice Liaison Officers (GPLOs) in Australia. Canberra: Commonwealth of Australia; 2002.

22. Bauer M. Content Analysis. An Introduction to its Methodology; By Klaus Krippendorff From Words to Numbers. Narrative, Data and Social Science; By Roberto Franzosi. British Journal of Sociology 2007; 58(2):329-31.

23. NSW Health. NSW Integrated Perinatal and infant Care (IPC) Strategic Framework for Mental Health. Sydney: NSW Department of Health; 2006.

24. NSW Health. Maternal and Child Health Home Visiting Policy - Primary Health Care. Sydney: NSW Department of Health; 2008.

25. ACT Health. Maternal and Child Health Support for Families. Canberra: ACT Department of Health; 2007. 
26. Kemp L, Eisbacher L, McIntyre L, O'Sullivan K, Taylor J, Clark T, et al. Working in partnership in the antenatal period: What do child and family health nurses do? Contemporary Nurse 2006; 23(2):312-20.

27. Briggs C. Nursing Practice in Community Child Health: developing the nurse-client relationship. Contemporary Nurse 2006; 23(2):303-11.

28. Kruske S, Barclay L, Schmied V. Primary health care, partnership and polemic: child and family health nursing support in early parenting. Australian Journal of Primary Health 2006;12(2):5765.

29. Talbot L, Verrinder G. (Eds) Promoting Health: The Primary Health Care Approach. Sydney: Elsevier; 2005.

30. Child \& Family Health Nurses Association. Competency standards for child and family health nurses. 2nd ed. Sydney: Child \& Family Health Nurses Assoc (NSW) Inc. Standards \& Practices Subcommittee, (NSW); 2000.

31. Foureur M. Next steps: public health in midwifery practice. In: O Luanaigh P, Carlson C, editors. Midwifery and Public Health: Future Directions and New Opportunities. London: Elsevier; 2005. p. 221-37.

32. ANMC. National Competency Standards for the Midwife. Canberra: Australian Nursing and Midwifery Council; 2006.

33. Saultz J. Defining and measuring interpersonal continuity of care. Annals of Family Medicine 2003;1(3):134-43.

34. Saultz J, Lochner J. Interpersonal continuity of care and care outcomes: a critical review. Annals of Family Medicine 2005;3(2):159-66.

35. McCourt C, Stevens T, Sandall J, Brodie P. Working with women: Developing continuity of care in practice. In: Page LA, McCandlish R, editors. The New Midwifery: Science and Sensitivity in Practice. 2nd ed. Philadelphia: Churchill Livingstone: Elsevier; 2006.

36. Leap N, Pairman S. Working in partnership. In: Pairman S, Pincombe J, Thorogood C, Tracey S, editors. Midwifery: Preparation for Practice. Sydney: Churchill Livingstone Elsevier; 2006. 
Table 1: Respondents by Area Health Service

\begin{tabular}{lc}
\hline Area Health Service in NSW & $\mathrm{N}(\%)$ \\
& $\mathrm{N}=67(100 \%)$ \\
\hline - Greater Southern & $16(24)$ \\
- Sydney South West & $14(21)$ \\
- Hunter New England & $10(15)$ \\
- Northern Sydney Central Coast & $8(12)$ \\
- North Coast & $7(10)$ \\
- Sydney West & $6(9)$ \\
- Greater Western & $3(4)$ \\
- The Children's Hospital & $2(3)$ \\
\hline
\end{tabular}


Table 2: Respondents by their stated role or position

\begin{tabular}{lc}
\hline Role & $\mathrm{N}(\%)$ \\
& $\mathrm{N}=67(100 \%)$ \\
\hline - Midwifery manager & $19(28)$ \\
- CFHNwifery consultant, outreach/community midwife & $14(21)$ \\
- CFHN* Nurse Consultant, clinical nurse specialist or educator & $12(18)$ \\
- Families First (now Families NSW) Coordinator & $13(19)$ \\
- Other & $4(6)$ \\
\hline
\end{tabular}

*Child and Family Health Nurse 
Figure 1: Continuity of care as a hierarchical concept [33,35]

\begin{tabular}{|c|c|c|}
\hline Level of continuity & Definition & $\begin{array}{l}\text { Considerations for effective } \\
\text { transition from midwifery to } \\
\text { CFHN }\end{array}$ \\
\hline $\begin{array}{l}\text { Informational } \\
\text { continuity }\end{array}$ & $\begin{array}{l}\text { readily available to any health } \\
\text { care professional caring for her } \\
\text { and can be accessed and } \\
\text { communicated among those } \\
\text { involved in the care. } \\
\text { Concept might be the most } \\
\text { important aspect of continuity in } \\
\text { preventing medical errors and } \\
\text { ensuring safety, but by itself } \\
\text { informational continuity might } \\
\text { not improve access to, or } \\
\text { experience of, care. }\end{array}$ & $\begin{array}{l}\text { - Systems exist to ensure } \\
\text { information is carried from } \\
\text { one provider to another. } \\
\text { - This includes } \\
\text { communication, } \\
\text { collaboration and IT } \\
\text { systems that assist care } \\
\text { providers through effective } \\
\text { transfer of information. }\end{array}$ \\
\hline $\begin{array}{l}\text { Longitudinal } \\
\text { continuity }\end{array}$ & $\begin{array}{l}\text { - creates a familiar setting in which } \\
\text { care can occur and should make it } \\
\text { when needed } \\
\text { - each woman or family has a } \\
\text { 'place' where she receives most } \\
\text { care and a team who assumes } \\
\text { responsibility for coordinating the } \\
\text { quality of care, including }\end{array}$ & $\begin{array}{l}\text { - A small group or team of } \\
\text { midwives work closely } \\
\text { with CFHNs to effectively } \\
\text { support the transition of } \\
\text { women and families. } \\
\text { - Services could also be co- } \\
\text { located in the community, } \\
\text { with shared management } \\
\text { structures, have a culture }\end{array}$ \\
\hline
\end{tabular}




\begin{tabular}{|c|c|c|}
\hline & preventive services. & $\begin{array}{l}\text { of collaboration with } \\
\text { processes that facilitate } \\
\text { dovetailing rather than } \\
\text { duplication. }\end{array}$ \\
\hline Relational continuity & $\begin{array}{l}\text { - the development of personal trust } \\
\text { between an individual care } \\
\text { provider and a recipient of care } \\
\text { - an ongoing relationship exists } \\
\text { between each woman and a care } \\
\text { provider } \\
\text { - the woman or family knows the } \\
\text { provider by name and has come } \\
\text { to trust them on a personal basis }\end{array}$ & $\begin{array}{l}\text { - Childbearing women have } \\
\text { a known midwife and a } \\
\text { known CFHN. } \\
\text { midwifery group practice } \\
\text { teams of CFHNs to } \\
\text { provide familiar care } \\
\text { providers who the women } \\
\text { trusted and who trusted } \\
\text { one another. }\end{array}$ \\
\hline
\end{tabular}

